\title{
Implantação da Comissão de Prevenção e Tratamento de Lesões na Pele em um Hospital Público
}

\section{Commission for Prevention and Treatment of Skin Injuries Implantation in a Public Hospital}

\author{
Roberta Carozo Torres ${ }^{1}$ \\ Sacha Jamille de Oliveira ${ }^{2}$
}

Ana Cristina Freire Abud $^{3}$

Rita Maria Viana Rego ${ }^{4}$

\footnotetext{
${ }^{1}$ Enfermeira. Mestra pelo Programa de Pós-Graduação em Enfermagem da Universidade Federal de Sergipe (UFS). Professora do Instituto Federal de Alagoas - IFAL. Membro da Liga Acadêmica em Estomaterapia de Sergipe - LAE/ UFS. Aracaju/ SE, Brasil. E-mail: robertacarozo@gmail.com. Autor correspondente.

${ }^{2}$ Enfermeira. Mestra pelo Programa de Pós-Graduação em Enfermagem da Universidade Federal de Sergipe (UFS). Especialista em Saúde do Adulto e do Idoso. Membro da Liga Acadêmica em Estomaterapia de Sergipe - LAE/ UFS. Aracaju/ SE, Brasil. E-mail: sachajamille@hotmail.com.

${ }^{3}$ Enfermeira. Doutora em Enfermagem pela Escola de Enfermagem de Ribeirão Preto (USP). Professora adjunta do Departamento de Enfermagem da Universidade Federal de Sergipe (UFS). Coordenadora da Liga Acadêmica em Estomaterapia de Sergipe - LAE/ UFS. Aracaju/ SE, Brasil. E-mail: acfabud@uol.com.br.

${ }^{4}$ Enfermeira. Doutora em Enfermagem pela Universidade Federal do Ceará (UFC). Professora colaboradora do Programa de Pós-graduação em Enfermagem da Universidade Federal de Sergipe (UFS). São Cristóvão/ SE, Brasil. E-mail: ritamvrego@gmail.com.
} 


\section{Resumo}

O cuidado aos indivíduos com lesões na pele é um desafio multiprofissional, porém com maior responsabilidade para a equipe de enfermagem, por ter a responsabilidade da execução dos curativos. Este estudo objetivou relatar a experiência de implantação da Comissão de Prevenção e Tratamento de Lesões na Pele (CPTLP) em um hospital público do estado de Sergipe. Trata-se de um estudo descritivo, do tipo relato de experiência, realizado a partir da vivência profissional na CPTLP de um hospital público de Sergipe. Com o intuito de sistematizar a assistência aos portadores de feridas e aqueles com risco de desenvolvê-las, a equipe da CPTLP foi criada e organizada com a finalidade de prevenir e tratar lesões na pele dos pacientes internados. A CPTLP tem o enfermeiro como profissional de referência para esses cuidados tendo em vista o protagonismo desse profissional nesse cenário. Assim, a equipe da CPTLP tem conseguido minimizar o surgimento de lesões complexas, além de tratar de forma efetiva as existentes através do atendimento individualizado e educação das equipes assistenciais.

Palavras-chave: Ferimentos e lesões; Cuidados de Enfermagem; Segurança do Paciente.

\section{Abstract}

Care for individuals with skin lesions is a multiprofessional challenge, but with greater responsibility for the nursing team, for having responsibility for the dressings execution. This study aimed to report the experience of the Commission for the Prevention and Treatment of Skin Injuries (CPTLP) implementation in a public hospital in the state of Sergipe. This is a descriptive study, of the experience report type, carried out from the professional experience in the CPTLP of a public hospital in Sergipe. To systematize assistance to wound patients and those at risk of developing them, the CPTLP team was created and organized to prevent and treat injuries to the patients' skin. The CPTLP has the nurse as a reference professional for such care in view of this professional role in this scenario. Thus, the CPTLP team has managed to minimize the complex lesions occurrence, in addition to effectively treating existing ones through the individualized care and the care teams' education.

Keywords: Wounds and Injuries; Nursing Care; Patient Safety. 


\section{Introdução}

As lesões de pele são consideradas um problema de saúde que pode acarretar dificuldades na vida de quem as possui, além de demandar um maior tempo de trabalho da equipe de enfermagem durante a assistência e aumentar o tempo de permanência do paciente na unidade hospitalar, o que contribui para uma elevação dos gastos com materiais e insumos ${ }^{(1)}$.

Cuidar de indivíduos com lesões na pele é um desafio multiprofissional, porém com maior responsabilidade para a equipe de enfermagem, pois é essa quem se encontra em contato direto com o paciente por um maior período de tempo e que geralmente fica incumbida da realização dos curativos, sendo importante destacar que para tal, a equipe de enfermagem deve estar munida de conhecimento específico, baseado em evidências científicas, que possam proporcionar a correta avaliação da lesão, a adequada execução da técnica de curativo e com isso, uma assistência de qualidade ${ }^{(2)}$.

Entretanto, esse cuidado deve ser realizado de forma integral, de modo a abordar o indivíduo como um ser biopsicossocial, ultrapassando a mera execução da técnica de curativo $^{(3)}$. Assim, a assistência de enfermagem ao portador de lesões na pele deve buscar promover a autoestima, autonomia e autocuidado, por meio de intervenções que busquem assistir às necessidades humanas integralmente, sem restringir-se a lesão(4).

Os cuidados com as lesões de pele, mais especificamente a prevenção do aparecimento dessas lesões, está inserido no contexto da segurança do paciente, que por sua vez, visa à diminuição de danos aos pacientes decorrentes de uma assistência inadequada, ao prevenir o acontecimento de erros e eventos adversos, garantindo assim a integridade do paciente e uma assistência de qualidade ${ }^{(5)}$.

A segurança do paciente é um tema debatido em âmbito mundial e que tem ganhado destaque e respaldo a partir dos vários estudos realizados, mas o que se pode observar é que mesmo com todo conhecimento existente sobre o tema, existem fatores que dificultam a realização plena de uma assistência segura como observado num estudo realizado em unidades de terapia intensiva no sudeste do Brasil, que destacou a relação entre a ocorrência de eventos adversos e a realocação da equipe de enfermagem, ao justificar que o maior número de eventos adversos se deveu a inadequada realocação dos profissionais, pois em alguns casos o profissional não possuía a experiência necessária para lidar com pacientes críticos ${ }^{(6)}$.

Dentre os eventos adversos considerados como prioridade na segurança do paciente estão as lesões por pressão (LPP), as quais acometem os pacientes hospitalizados que se encontram acamados, ou seja, que apresentam alguma alteração na mobilidade, sendo de suma importância que para o paciente nessa condição, além das medidas preventivas, seja levado em conta o estado nutricional e as comorbidades apresentadas pelo mesmo, fatores que podem interferir na evolução dessas lesões ${ }^{(5)}$. Portanto, a prevenção e o tratamento dessas lesões é um dos focos das comissões de cuidado com a pele existente nas unidades hospitalares.

Desta forma, visando a segurança do paciente e a realização de uma assistência sistematizada para os pacientes já com lesões na pele foi que surgiu a necessidade de implantação de uma Comissão de Prevenção e Tratamento de Lesões na Pele (CPTLP) em um hospital público de Sergipe, a fim de minimizar o surgimento de lesões e prestar assistência a todos os pacientes já acometidos por algum tipo de lesão, contemplando a avaliação de risco para desenvolvimento de lesão, como também o emprego do tratamento adequado e acompanhamento da evolução das existentes.

Diante disso, justifica-se a relevância do presente estudo ao pretender divulgar a importância da existência de uma equipe multidisciplinar para o cuidado sistematizado às lesões de pele dentro de uma instituição de saúde, a fim de melhorar a qualidade de vida do paciente.

Assim, este estudo tem por objetivo relatar a experiência de implantação da Comissão de Prevenção e Tratamento de Lesões na Pele (CPTLP) em um hospital público do estado de Sergipe. 


\section{Método}

Trata-se de um estudo descritivo, do tipo relato de experiência, realizado a partir da vivência profissional de uma das autoras enquanto enfermeira da Comissão de Prevenção e Tratamento de Lesões na Pele (CPTLP) de um hospital público do estado de Sergipe entre 2016 e 2017.

O estado de Sergipe, situado no nordeste, é o menor dos estados brasileiros abrigando uma população estimada de 2.288.116 habitantes, composto por 75 municípios( ${ }^{(7)}$, e divide-se em sete regiões de saúde ${ }^{(8)}$.

A rede hospitalar é composta por 58 hospitais e 3596 leitos distribuídos nas regionais de saúde, destes 31 são privados, sendo $10 \mathrm{com}$ fins lucrativos e 21 privados sem fins lucrativos; 27 públicos, sendo dois federais, oito estaduais e 17 municipais. A região de Aracaju por ser a mais populosa e concentrar grande parte dos equipamentos e serviços de alta complexidade, é considerada como referência para as demais ${ }^{(8)}$.

\section{Resultados e Discussão \\ Contextualização sociocultural da instituição}

O Hospital de Urgência de Sergipe (HUSE) encontra-se situado na capital do estado, Aracaju, que está localizada no litoral, ocupa uma área de $181,8 \mathrm{~km}^{2}$, possui uma população de 650.106 habitantes ${ }^{(7)}$.

O HUSE possui 596 leitos, e por se tratar de um hospital de alta complexidade, especializado em atendimentos de urgência e emergência que é referência em trauma, recebe pacientes com os mais variados tipos de lesões de pele. São atendidos não apenas pacientes com afecções traumáticas, mas também clínicas, o que leva a uma variedade de feridas, desde as mais simples até as mais complexas.

Diante desse quadro de complexidade, em 2012 surgiu a necessidade de ter uma equipe especializada para acompanhar a evolução desses ferimentos, surgindo então a Comissão de Feridas. Na rede hospitalar estadual é a instituição pioneira a possuir uma equipe especializada em tratamento de feridas, além desta, a maternidade estadual também conta com uma equipe.

\section{O cuidado ao portador de lesões na pele}

No início essa comissão era composta por apenas uma enfermeira e uma técnica de enfermagem que eram responsáveis por avaliar e acompanhar as feridas de maior complexidade, por demanda espontânea, conforme solicitação da equipe médica.

Diante do trabalho desenvolvido, do aumento da demanda e da necessidade de reforçar as ações relacionadas à segurança do paciente, com o objetivo de melhorar a assistência, principalmente no que tange à prevenção das LPPs, foi criada então a Comissão de Prevenção e Tratamento de Lesões na Pele (CPTLP) em 2015.

Esta Comissão é composta por uma equipe multidisciplinar (fisioterapeuta, enfermeiros, técnicos e auxiliares de enfermagem) que atua realizando busca ativa diária de pacientes com risco de desenvolver lesões na pele e portadores de lesões, qualquer que seja a etiologia.

Além dessa busca ativa, o enfermeiro da CPTLP acompanha as lesões mais complexas executando o curativo diariamente, de modo a dar suporte às equipes assistenciais nesse tipo de cuidado. O enfermeiro é o responsável pela avaliação diária e sugestão da melhor cobertura para cada tipo de ferida e prescrição dos cuidados de enfermagem para com a pele.

Para tanto, a atuação do enfermeiro deve estar fundamentada na enfermagem baseada em evidências, pois esta auxilia o planejamento e execução de uma assistência adequada e de qualidade. Exige do profissional constante busca de conhecimento por meio de pesquisas, estudos e uso de novas tecnologias a fim de tornar-se capaz de decidir, em conjunto com o paciente e a equipe, qual o melhor tratamento para uma determinada lesão(9).

Inicialmente, os atendimentos da equipe da CPTLP consistiam em avaliações de pacientes com lesões complexas que não evoluíam. Essas avaliações eram realizadas por meio de solicitações informais das equipes de enfermagem e médica.

Com o intuito de sistematizar a assistência aos portadores de feridas e aqueles com risco de desenvolvê-las, a equipe da CPTLP buscou organizar-se estruturando suas ações a partir de um regimento e da elaboração de uma ferramenta que auxiliasse 0 acompanhamento desses 
pacientes de modo sistematizado, com base nas recomendações encontradas em literatura nacional e internacional.

Esse regimento foi elaborado pela equipe da CPTLP em conjunto com a Direção de Enfermagem a partir do modelo de outros regimentos da instituição, e regulamenta as finalidades, composição da CPTLP e atribuições de seus membros.

\section{Atuação da Comissão de Prevenção e Tratamento de Lesões na Pele}

Tendo em vista a efetivação das ações voltadas para a segurança do paciente, definiu-se como prioridade a prevenção das lesões de pele (em especial as lesões por pressão) e a educação em serviço das equipes assistenciais a fim de otimizar a assistência e melhorar a qualidade de vida dos pacientes.

Determinou-se então que as avaliações e acompanhamentos de pacientes com lesões seriam feitos a partir de solicitações registradas em prontuário pela equipe médica, e comunicadas à CPTLP pela equipe de enfermagem de cada unidade de produção.

A CPTLP tem promovido diversas capacitações para os seus membros, bem como para a equipe assistencial por meio de cursos específicos sobre desbridamentos, curativos e coberturas, prevenção de lesões de pele, dentre outros, por meio de reuniões científicas realizadas trimestralmente, e ações bimestrais de educação continuada. Tem buscado também desenvolver pesquisa na área e publicação de casos a fim de divulgar o trabalho desenvolvido e proporcionar conhecimento para os profissionais.

Anualmente a CPTLP realiza um grande evento aberto ao público em alusão ao Dia Mundial de Prevenção de Lesão por Pressão, onde são abordadas ações e estratégias de prevenção, além da apresentação de indicadores e casos acompanhados pela CPTLP. Desde a criação da CPTLP tem-se observado uma melhora na assistência de enfermagem no que diz respeito às técnicas adequadas de curativos e prevenção de LPP.

Dentre as dificuldades enfrentadas pela equipe da CPTLP, o suprimento das coberturas é o que mais afeta a assistência prestada. Por vezes ocorre o desabastecimento, que gera descontinuidade do tratamento e prolonga o tempo de internação, além de aumentar os gastos hospitalares.

Além disso, a falta de nutricionista e médico cirurgião (plástico e/ou geral) exclusivos na equipe da CPTLP por vezes dificulta o planejamento da assistência multiprofissional, visto que os plantonistas priorizam os atendimentos de acordo com as demandas da instituição, e por vezes quando solicitados pela CPTLP demoram a avaliar os pacientes.

\section{Conclusão}

A atuação de uma equipe completa, multi e interdisciplinar no tratamento das lesões de pele é necessária e traz excelentes resultados. Otimizar o cuidado aos pacientes com feridas e evitar que os mesmos as adquira durante o tratamento hospitalar diminui o tempo de permanência na unidade e proporciona uma melhor qualidade de vida aos pacientes.

A busca pela assistência ao ser humano de modo integral, conforme preconiza o Sistema Único de Saúde, requer dos profissionais o acolhimento permanente da pessoa para que, apesar da convivência com as lesões, ela consiga manter a sua qualidade de vida.

A CPTLP ainda não conta com profissionais de extrema importância no processo de cuidado aos portadores de lesões como o nutricionista e o cirurgião (plástico e/ou geral). Atualmente quando se faz necessária a avaliação destes profissionais ela é feita ao plantonista do dia, o que gera de certa forma uma descontinuidade da assistência.

Assim, o enfermeiro tem um papel fundamental nesse processo por ser o profissional que acompanha diariamente a evolução do paciente, pois firma com este um vínculo precioso para o cuidado, sendo o responsável pela sugestão e acompanhamento da terapêutica, o que tem fortalecido e dado autonomia à categoria dentro da instituição.

\section{Referências}

1. Krause TCC, Assis GM, Danski MTR. Implantação de uma Comissão de Cuidados com a Pele em um Hospital de Ensino. Rev Estima 
[Internet]. 2016 [citado em 18 abr 2017];14(1):13-20. Disponível em: https:// www.revistaestima.com.br/index.php/esti ma/ article/download/115/pdf.

2. Martins NA, Sena CA, Alves MS, Moura DCA. Teoria de intersistemas de Bárbara Artinian na consulta de enfermagem junto à pessoa com lesão de pele. CuidArte Enfermagem [Internet]. 2015 [citado em 18 abr 2017]; 9(1):09-17. Disponível em: www.fundacaopadrealbino.org.br/ facfipa/ner/pdf /Revistacuidarteenfermagem\%20v. \%209\%20n.1 20\%20jan.\%20jun\%202015.pdf.

3. Faria GBG, Prado TN, Lima EFA, Rogenski $M B$, Borghdt AT, Massaroni L. Conhecimento e prática dos enfermeiros sobre o cuidado com feridas. Rev Enferm UFPE on line [Internet]. 2016 [citado em 20 abr 2017]; 10(12):4532-8. Disponível em:

www.revista.ufpe.br/revistaenfermagem/index.ph p/revista/article/viewFile/9885/pdf_1829.

4. Bedin LF, Busanello J, Sehnem GD, Silva FM, Poll MA. Strategies to promote self-esteem, autonomy and self-care practices for people with chronic wounds. Rev Gaúcha Enferm [Internet]. 2014 [citado em 23 abr 2017]; 35(3):61-7. Disponível em: http://www.scielo.br/scielo.php? script=sci_arttext\&pid=S198314472014000300061 \&lng=en\&nrm=i so.

5. Ministério da Saúde (BR). Agência Nacional de Vigilância Sanitária. Manual segurança do paciente e qualidade em serviços de saúde: uma reflexão teórica aplicada à prática. Brasília: Ministério da Saúde, 2013 [citado em 27 jul 2017]. Disponível em: https://www20.anvisa.gov.br/ segurancadopacient e/images/documentos/livros/ Livro1-Assistencia_Segura.pdf.

6. Gonçalves LA, Andolhe R, Oliveira EM, Barbosa RL, Faro ACM, Gallotti RMD, et al. Alocação da equipe de enfermagem e ocorrência de eventos adversos/incidentes em unidade de terapia intensiva. Rev Esc Enferm USP [Internet]. 2012 [citado em 08 ago 2017]; 46(Esp):71-7. Disponível em: http://www.scielo.br/pdf/reeusp/ v46nspe/11.pdf.

7. Instituto Brasileiro de Geografia e Estatística. IBGE Cidades, 2017.
8. Funesa. Atenção Hospitalar no Estado de Sergipe - saberes e tecnologias para implantação de uma política. Livro do aprendiz 3/ Fundação Estadual de Saúde - Secretaria de Estado da Saúde de Sergipe. Aracaju: FUNESA, 2012.

9. Brum MLB, Poltronieri A, Adamy EK, Krauzer IM, Schmitt MD. Protocolo de assistência de enfermagem a pessoas com feridas como instrumento para autonomia profissional. Rev Enferm UFSM [Internet]. 2015 [citado em 18 abr 2017]; 5(1):50-7. Disponível em:

www.scielo.org.co/scielo.php?script=sci_arttext\& pid $=$ S0120-53072016000200019. 